\title{
Applications of Extended Reality Technologies within Design Pedagogy: A Case Study in Architectural Science
}

\author{
Vincent Hui, Tatiana Estrina, Gloria Zhou, Alvin Huang \\ Ryerson University, Canada
}

\begin{abstract}
The concept of virtualizing and augmenting realities through technology has evolved from fantasy to feasibility and has advanced how humans are able to visualize and interact with the digital world. Extended realities (XR) often interpret threedimensional space in both realistic and conceptual forms, leveraging the ability of macro and micro scaling of computerized images. The versatility of $V R$ is used in a wide range of disciplines from creative industries to professional practices and as an interactive multi-sensory visualization medium, it can be effectively adopted as a learning tool, used to elevate the experience in the classroom. This paper examines the possibilities of the incorporation of virtual reality, augmented reality, and mixed reality into the post-secondary architectural academic setting through lecture-based education, design pedagogy, project feedback delivery, and enhancement of experiential learning. The paper provides a case study of implementation into models of pedagogy at Canada's largest architecture program, in order to enhance the learning experience both within in-person and online learning contexts.
\end{abstract}

\section{Introduction}

These While much of post-secondary education involves understanding situations that either do not exist or are remote from the classroom, XR technologies provide the opportunity for the students to gain a greater understanding of such concepts through digital experience. Although in mainstream culture, XR has been primarily used in the context of entertainment, the technology has also been applied to a multitude of professional industries including archeology, sciences, architecture and engineering. [1]

The integration of XR into pedagogy provides an opportunity for a new paradigm to emerge, one which prioritizes experiential learning as a primary means of education. Such a shift has regained new traction in recent months, as changes in course delivery to online learning caused by the COVID-19 pandemic outbreak has elicited a renewed interest in the reexamination of digital tools in pedagogy. This paper presents a case study of the implementation of XRs across the undergraduate curriculum at Ryerson
University's Department of Architectural Science (DAS). Three XR technologies were adapted into numerous facets of architectural instruction in order to enhance theoretical content, increase accessibility to learning, improve visualization of design work, and heighten student digital savvy. The multitude of techniques and opportunities for implementation are framed within the context of lecture-based instruction, design pedagogy, project feedback delivery, and experiential learning.

\section{Overview of Extended Reality Typologies}

Extended Reality (XR) is a culmination of the spectrum of immersive realities including VR, AR, and MR. XR becomes an extension of the physical environment for users through using human-machine interactions generated by computer and wearable technology, further blurring the lines between reality and the virtual world to become indistinguishable.

\subsection{Virtual Reality}

Virtual reality (VR) is an emerging technology that allows for "the user to interact with a computergenerated three-dimensional model or virtual environment" through the placement of a screen or headset over the eyes [1]. Although popularized in the 1990s, recent technological developments allowed for VR to not only permeate into mainstream culture but also education [2].

VR experiences manifest themselves in a multitude of different ways, most notably through 360-degree still VRs, which provide a stereoscopic view of an image or video for the viewer, navigable VRs which give the viewer the opportunity to travel within the virtual environment, and interactive VRs which allow for the user to create within a virtual space they occupy.

At its inception, VR headsets were expensive recreational devices that were typically inaccessible, difficult to install, and required a very powerful desktop to operate. However, recently headsets such as the Oculus Quest, have been made available at a fraction of the cost [2]. In addition, mobile developments have made phone devices capable of 
displaying VR environments when augmented by headset enclosures, such as the Google Cardboard. These headsets are very affordable, easily distributable, and accessible for students. This made navigable VR implementation begin to permeate into the mainstream culture as well as pedagogy.

\subsection{Augmented Reality}

While VR's purpose is to immerse the user into an entirely different environment, augmented reality (AR) uses screen overlays to incorporate digital objects within the physical environment. Although generally unable to react to changes in the space, AR technologies are able to layer digital information over the physical. AR rose to mainstream popularity through its use in Pokémon Go and a number of other mobile applications. Although previously the combination of webcam and computer displays were used for AR, modern mobile phones are now also able to successfully act as vehicles for AR experiences. [3]

\subsection{Mixed Reality}

An extension of AR technologies, mixed reality (MR) also serves as a means to digitally augment the user's physical environment. However, MR goes beyond a simple overlay and instead becomes a true bridge between the physical and the digital, computationally responding to changes in the user's context. Thus, mixed reality is not only an independent overlay, but instead is able to recognize and interact with physicality. The most expensive to engage with of the three extended realities discussed, MR state-of-the-art headsets, such as the Leap Motion or Microsoft's HoloLens 2, display holographic imagery over users' environments. These devices provide head tracking, recognition of QR codes, and are able to be controlled via and gestures, making their use entirely handsfree [3]. Despite the technology still being in its nascency, interest, research, and use of MR has been on the rise in recent years, especially in the AEC industry.

\section{Extended Realties within Architectural Education}

Extended realities in architecture have developed immensely within the construction industry from aiding in design development to assisting in assembly during construction of building projects. In addition, the integration of XR in architectural pedagogy has emerged within experiential learning applications, proving to be a tool that further extends the possibilities of design and visualization in the classroom.
VR has found a large and growing market within the AEC industry. As architecture takes years and millions of dollars to construct yet is very difficult to understand spatially until it is built, immersive technologies like VR are able to aid in visualization of spaces. Where orthographic and 2D drawings are difficult for laypeople to understand spatially, being able to digitally occupy the space aids in the communication and improves project delivery. The ability to occupy spaces is also very beneficial for designers, as they are able to view their spaces in three dimensions and enhance their design work and process. In the context of academia, students are aided by such digital augmentation as they can better understand their spaces and design implications in three dimensions.

Navigable VR platforms have become more common in serving a multitude of purposes such as training and educating professionals to perform medical and technological tasks as well as construction. Such applications have proven to be very effective in training for both operation of machinery and more specified tasks [4]. In the field of archaeology, VR is used frequently for the representation of both archaeological sites and reconstruction of historical architecture for the purposes of dissemination to students and the general public [5]. The interactive capabilities in VR have become popular as a new way to play video games, allowing players to utilize their full body in gameplay and become immersed in the digital realm. Although interactive VR environments have grown viable in the entertainment industry, it has also found a purpose in the academic and professional fields. For example, applications of VR in the medical field have been recognized as a learning tool through its ability to be highly interactive, digitally generated, and emulating the impression of presence. Ultimately, to give users an opportunity to understand and visualize elements of the real world that otherwise may not be comprehensible. Interactive VR is also effective in the creative fields, yielding the freedom of expression and experimentation in a seemingly infinite digital space. The plethora of uses for interactive VR environments is evident in fields ranging from professional to creative, showcasing its practicality and ease of use. However, interactivity can also prove to be suitable in education as an immersive apparatus for teaching.

AR technologies have less applications within the AEC industry as many previous uses have been replaced by the more elaborate MR technology. However, they are often used as an overlay of information over top site or building contexts. Largely a visual aid, AR is able to supplement physical environments with additional unbuilt digital information. Although still in its nascency, applications such as Trimble advancing the potential of AR technology in the industry. Some examples of 
software that support this workflow include AR Workbench, and ARTHUR which are experimented as augmentation and manipulation tools to impose digital models onto physical sites [6] ARTHUR's platform uses gestural cues to allow students to generate simulations of their architectural 3D model. While currently the uses of VR and AR are largely confined to representation applications within the AEC industry, MR has been able to surpass visualization. Via headsets, the architecture industry has been able to guide construction and fabrication processes through augmentation. A number of construction projects have been able to engage with MR, such as the bricklaying project in Tasmania, which involved skilled laborers in the construction of a parametrically complex wall. In this project, the technology served as 2D guidelines for the placement of the 3D objects, namely bricks, in order to reduce the need for complex construction documentation and on-site measurements and markings. In addition, due to the enhanced capabilities of MR, the software was able to recognize the geometries of the bricks and provide live feedback to the laborers on the accuracy of the brick placement [7].

In parallel to using MR as guide work for fabrication and construction, the technologies have also been used for the education and training of skilled labor trainees. These technologies eliminate much of the confusion and complexity of drawings and measurements to focus on the actual crafts processes. MR has been used both in training for machinery operation, where the technology is able to indicate and guide the use of the machinery, and skilled craft, using the holographic guide forms. For instance, in the bricklaying project, an experienced bricklayer worked with a trainee, both equipped with MR headsets. As both were able to see the holographic guides and the live feedback, the mentor capitalized on the technology to instruct the trainee in the construction process [7].

Table 1. Summary of XR applications within the undergraduate curriculum at DAS

\begin{tabular}{|l|l|l|}
\hline Pedagogical Context & XR Typology & Applications \\
\hline Extended Realities for & VR & Interactive Virtual Field Trips (iVFTs) \\
\cline { 2 - 3 } Lecture-Based Instruction & AR & GPS-based augmented learning \\
\hline $\begin{array}{l}\text { Extended Realities for Design } \\
\text { Ideation }\end{array}$ & VR & Student-generated navigable environments \\
\cline { 2 - 3 } Extended Realities for Design & AR & Representation of designs within site conditions \\
\cline { 2 - 3 } Feedback & VR/AR/MR & Social and occupiable environments \\
\hline $\begin{array}{l}\text { Extended Realities for } \\
\text { Experiential Learning }\end{array}$ & VR & Digital interactive meetings \\
\cline { 2 - 3 } & MR & Virtually augmented fabrication \\
\hline
\end{tabular}

Within architectural pedagogy at the DAS, applications of the three XR typologies are outlined in Table 1 . VR is largely used for immersion into both previously built work and student designs, aiding in visualization. Meanwhile, AR is applied in on-site contexts, in order to provide and represent additional design information. MR has been implemented into fabrication, as holographic guide work for the assembly of complex student-designed geometries

\section{Extended Realities for Lecture-Based Instruction}

The traditional architectural education largely emphasizes visual and verbal communication, it is frequently inadequate for a well-rounded understanding of the subject matter. Engagement through emerging technologies should be explored within and outside of classroom settings to build a stronger foundation for contextual understanding and analyses of course content. Applications of extended realities may also be an asset in history and theorybased lecture courses where students can observe details and virtualize site visits as an alternative to $2 \mathrm{D}$ media forms.
Our current period of increasing economic disparities has profound impacts on the programmatic abilities of institutions and educators, such that limitations may be imposed on potential resources [8]. While students are obtaining information from the same curricula, their access to basic equipment, such as computing and Internet connection, vary drastically due to their financial capabilities. Ultimately, educational opportunities such as fieldtrips and hands-on learning are impacted and limited to fewer students [2]. Technology must then be adapted and innovated within the program to accommodate for experiential learning through accessible and cost-effective methods. VR technology provides an alternative solution for many students and educators to virtualize experiences without the burden of high costs. Its reasonable fidelity and immersive nature are effective in developing an understanding of conceptualized and existing spaces. This is evident through many different types of VR environments, however, 360degree still photography offers a seamless transition from traditional visualization methods as it is easily accessible through readily available resources. Canada's largest architectural program has applied 
360-degree stills for the purpose of interactive virtual field trips (iVFTs) and conceptual visualizations (Figure 1).

The use of iVFTs as a learning tool has become essential in substituting the experience of site visits and/or travelling in different cities. While it is still far removed from physically visiting a locale, its effectiveness in capturing the experience is much greater than that of imagery, videos, and text available online [9]. Furthermore, it has been a necessitated medium for exploring spatial conditions and environments during the COVID-19 pandemic while students and educators are limited to remote learning [10]. VR content can be easily generated using consumer-grade cameras capable of capturing a 360-degree frame of view [2]. Using multi-media layering and motion sensors, the viewer may also navigate the space as if they were placed inside of it. At DAS, the Kultour learning experience offers students the opportunity to engage with the urban landscapes of destinations around the world using 360-degree stills and videos captured with this method (Figure 1). In addition, 360-degree VR content is also readily viewed, edited, and broadcasted using mobile hardware and software which makes it increasingly accessible when combined with the ubiquity of smartphones. Basic VR experiences such as iVFTs offer effective alternatives to site visits while also leveraging the vast amount of information and data readily available online.

Navigable environments have been used frequently for the representation of lost or historical architecture, but most notably in a museum installation titled "Archaeology Alive!", at the Whitchurch-Stouffville Museum just outside of Toronto. William Michael Carter, a professor of digital media at Ryerson University expanded his research for his $\mathrm{PhD}$ dissertation by inviting groups of students from architecture, video game design, and sound design to collaborate on an inter-institutional, interdisciplinary, multi-sensory project, re-creating a traditional Huron-Wendat longhouse (Figure 1). The navigable VR component was supplemented by a physical enclosure which stimulated the non-visual senses, such as the olfactory and the auditory. This award-winning display was not only able to help the local community better understand their history, architecture, and culture but also help students from the various disciplines to gain a greater understanding of the historical context.

$\mathrm{AR}$ is an invaluable tool which serves a multitude of purposes in lecture-based learning courses ranging from construction detailing and structures, to theory and history. The Arch-App, developed at DAS, offers an AR platform that provides "Geo-located data atop real-world displays on mobile devices", superimposing 3D models to conduct self-guided tours of Toronto [11]. Moreover, the app can perform as a database to communicate information about the existing contexts such as its historical background, dates of completion, and building architects. Students are then able to access data and insight through devices on hand that they may not be able to attain otherwise [11].
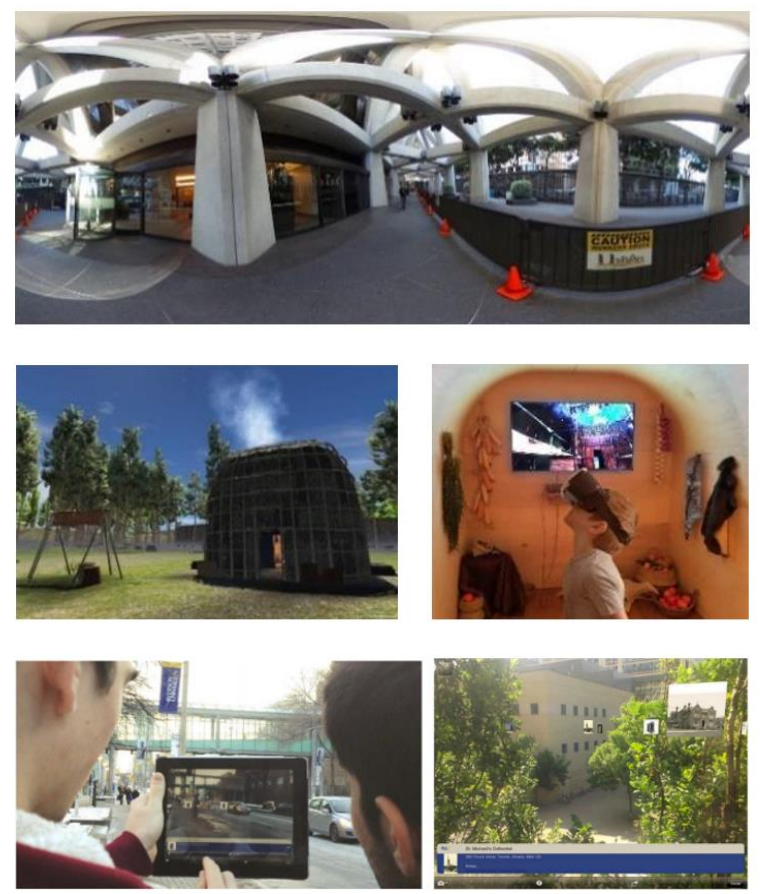

Figure 1. 360-degree still of building in San

Francisco captured for the purpose of iVFTs (above), the Archaeology Alive installation depicting a Huron-Wendat Longhouse (center), ArchApp in use (below)

The platform is not only effective in collecting and circulating information, but also an effective representation tool for students. Augmented reality can be used for an array of architectural purposes beyond database and representation applications. In this case, the Arch-App can be further applied as a tool for the digital archiving of architecture that has undergone demolition and exists as ruins. This information can then be used to layer on top of existing urban environments to juxtapose the different conditions. Additionally, the Arch-App uses media sources such as imagery and videos of buildings in different time periods and past construction, and orthographic drawings beyond models and texts to capture a greater scope of data. In lecture-based learning, extended realities are assets in architectural data collection, generating visualizations of both existing and historical sites, construction detailing, as well as providing costeffective alternatives to site visits. Its adaptable workflows coupled with its accessibility through smart devices encourages a seamless technological transition into lecture-based settings. 


\section{Extended Realities for Design Ideation}

The XR technology has been used to transport viewers into existing environments, its purpose can be extended to visualize conceptual designs using 3D models. Many students studying architecture are experienced with designing spaces via 3D modelling software as well as creating visualizations using rendering software. In most cases, 360-degree stills follow similar workflows to generate imagery such that they use the same programs and/or extensions into existing ones. It serves as an invaluable design tool during the iterative design stages as well as final presentation due to its ability to generate images quickly while also informing students of spatial relationships between its users and context.

In 2016, Yulio, a VR rendering software, was integrated into the studio curriculum at DAS [2]. Its introduction was seamless due to its compatibility with students' modeling software thus encouraging students to adopt this method of representation without many challenges (Figure. 2). Students could then superimpose their rendered 3D models onto site, which was captured using similar methods to the iVFTs, to communicate their concepts through an immersive experience. It has allowed students to become "more aware of the 3-dimensional character," as well as identify discrepancies within their models [12]. Students were readily able to adopt the medium in their design workflow; surprisingly, the institution was the first in Canada to implement $\mathrm{VR}$ in the core undergraduate architecture curriculum. Since then, development and research into VR technology has grown exponentially, allowing for multiple options of low-cost and intuitive rendering software to emerge, such as Twinmotion, Enscape, and V-Ray.
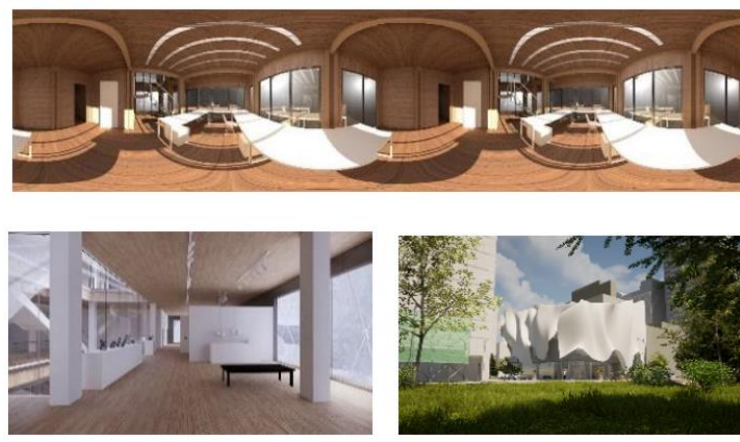

Figure 2. Studio project rendered as a 360-degree still VR image (above), Still views from a student's navigable Enscape VR walkthrough model (below)

Interactive worlds provide the users the full experience of occupying a location outside of one's physical environment. Where static VRs provide users with a VR photograph or video experience, navigable VRs allow the viewer to experience a $3 \mathrm{D}$ modeled space within their headset. As such the viewer is able to navigate through the space, having control of not only their placement within the space but also their vantage point. Such experiences generally transcend the solely visual stimulation still VRs provide, and allow for multisensory experience, such as auditory feedback. Due to the presence of the controller's vibrations, touch response allows for the telepresence to become much more realistic for the user [1]. In addition, platforms that support navigable VR experiences are also able to be operated via desktop interface, employing a similar navigation system to video games. Such an implementation allows for those unable to gain access to headsets an opportunity to participate without much additional investment.

Within architectural pedagogy, VR has begun to permeate into $3 \mathrm{D}$ visualization techniques as a means to create not only a static image but rather an environment that the viewer can occupy and interact with (Figure. 3). Various gaming engines have been optimized to generate navigable environments from 3D models that students create, where the user is able to have a live feed of the model as it is changed in real-time while also being able to view smaller details such as the undersides of tables and understand the progression of space [2]. In addition, such visualization methods allow for the control of elements such as sunlight during the time of day and auditory elements.

In addition to the experiences generated using VR as a visualization tool, it also prevails as an interactive method for designing in 3D space. Rather than using just the headset as a panoramic viewer, VR can be a more immersive experience through the inclusion of input devices, i.e., controllers, and spatial tracking to allow users to control how and where they move within the virtual environment and enables interfacing with $3 \mathrm{D}$ objects.

As a learning engine, VR can also apply in the classroom of architecture. Traditionally, studio course work is done through drafting and model making, with a popular integration of 3D modelling software now widely utilized. All strategies work towards materializing the concepts from the students' mind into a tangible form to be presented. In many ways, these strategies try to grasp the spatial qualities of a design, using scaled methods such as model making to build something similar to the design in hopes to experience it. However, with VR, designers can load their 3D model, whether still in a development stage, to design in a digital environment while interchangeably experiencing their designs simultaneously. Moreover, design can start from scratch within the VR space and can be translated to common 3D modelling software for further development in their studio pipeline. MindeskVR is a tool currently used within architectural pedagogy as a VR modelling tool compatible with commonly used 
3D modeling software and makes use of similar modelling commands as the native program, thus making this software very intuitive for the students. In addition, MindeskVR allows users to interface with their existing 3D model in collaboration with others, giving students the ability to engage with their peers and faculty while generating digital content (see Figure. 3).
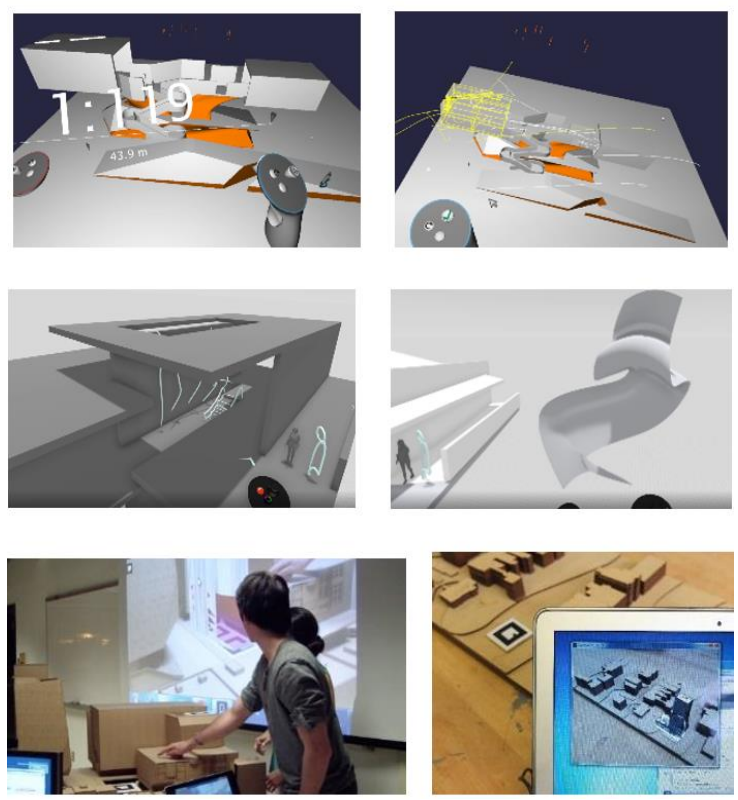

Figure 3. Students using MindeskVR in their modeling workflow (above), Students using Gravity

Sketch VR in their modeling workflow (center), $A R I D D$ used for design ideation (below)

Gravity Sketch VR is another VR modelling tool used within the architectural workflow. In contrast to the aforementioned MindeskVR software, it operates as a native program with its own tools and requires models to be imported for editing (see Figure. 3 ). The program also allows for multi-user creation, allowing for multiple designers to model in the same virtual space at the same time with real time feedback. The collaboration and intuitive model manipulation are reminiscent of group model making within architectural studios.

The modelling tools implemented in VR are intuitive to the user, emphasizing the use of ones' hands to manipulate digital models, mimicking a designer's interaction with physical scale models, while leveraging on the digital benefits of computer aided design such as real time interaction with parametric tools, and infinite possibilities within the digital realm. The simulated interaction has allowed designers to be timely conscious of design discrepancies in early stages of their design and allows for an immersive experience within the detailed design phases later [13]. The VR environments can also be given to others to interact with so designers can analyze how people occupy and operate within their designed space and to catch spatial faults within their project, all of which would have been very difficult to experience and to receive feedback from mere drawings and scaled down models.

It is critical for students to be able to visualize and conceptualize their designs in larger contexts where site influences can be evaluated and developed upon. Design ideation within existing contexts permits interactive design processes to take place where students can adopt AR to iterate ideas on a given site. Various AR platforms provide students with the advantage of viewing larger physical site conditions as well as visualizing factors that are intangible such as "wind speed, shadow movements, and traffic flow," to communicate with peers and faculty [6]. The Augmented Reality in Design Development (ARIDD) Project was developed at Ryerson University to "bridge the disconnect between design ideation and application in real work and real time," [14]. Students studying architecture are able to leverage the technology commonly used in the entertainment industry to rapidly generate iterations based on contextual suitability. Students can then superimpose their designs onto various media which can range from site to real-life conditions. In its application, the $A R I D D$ project has been introduced into Ryerson University's DAS core studio courses where students adopt this method of visualization and design ideation as a cost-effective alternative to model-making and efficient means of producing easily modifiable iterations. Additionally, it provides visual learners with additional ways of understanding classroom material. Moreover, utilizing AR in the $A R I D D$ Project fosters experiential learning opportunities as it satisfies the many facets of experiential learning as described by Jane Henry [14].

The applications of static 360-degree experiences, and the implementation of a number of software, such as Mindesk VR, Gravity Sketch, and ARIDD, students are given greater flexibility in design ideation and communication. It effectively communicates student ideas through non-traditional forms of visualizations and technology. Additionally, it is an asset in rendering environmental factors that are otherwise difficult to capture through orthographic drawings, and models. Students are able to leverage the platforms' ability to rapidly modify designs, visualize site contexts, and observe design elements in the project.

\section{Extended Realities for Design Feedback}

Within design education, the importance of feedback and comments are crucial in developing designs with different perspectives and revealing 
flaws whilst capturing opportunities that will push a design to grow. In architectural education, design reviews are common in analyzing buildings for both conceptual aspects such as connections to the site, massing composition, expression of materiality, and for technical aspects such as building code requirements, and dimensional constraints.

As a result of the COVID-19 pandemic, social separation has become a large barrier within design studios [10]. Furthermore, communication has been reduced to a single speaker video call, reaching a stagnant spotlight format restraining the intermingling found in a conventional studio setting, where students can organically converse about their projects, provide feedback and suggestions with the use of sketch models and drawings. However, multiuser VR has re-enabled these moments of interaction while incorporating the benefits of being within a digital space. VR meeting software allows for not only multi-user interaction and conversation, but also sharing of $2 \mathrm{D}$ and $3 \mathrm{D}$ media. These environments have become commonly used within professional contexts as an alternative for video conferencing. However, due to the newfound ability for a more stimulated and enhanced interaction, such platforms can also enrich academic discourse. With VR meeting platforms like Spatial.io, students and faculty are able to meet in a digital 'room' where digital drawings can be pinned up on a wall, inprogress models can be imported, and interaction between users is possible. Like a traditional studio, users can pick up a model or drawing, and attach notes of feedback, create a digital markup, and creators can save their critique comments to be revisited later, capturing every detail of design critique (see Figure. 4). In addition to the positive social benefits of multi-user VR, a sense of scale can be brought to a student's design discussion, where CAD models can be scaled to sizes where detail can be examined by an audience in a setting much more immersive than a 2-dimensional screen where impressions of dimensions and scale can be misinterpreted.
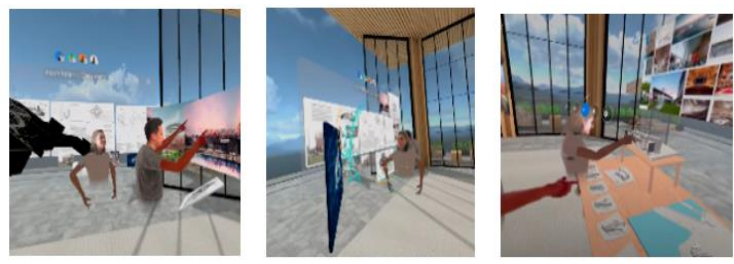

Figure 4. A virtual critique in Spatial.io, with a professor and students examining a project

Users are not required to be on the same network to be connected, therefore international meetings become viable and time saving. Thus, design feedback sessions are not limited to only the professors and students but also guest critiques from across the world are able to join in the digital space and provide different perspectives further enriching the learning experience for the students.

In addition, Spatial.io is also inclusive to those who do not have access to VR headsets and integrates video calling on web browsers and on mobile devices, including anyone inside the digital room. The AR capabilities using Spatial.io can be found in using mobile device cameras to project images and models into a user's room, allowing users to pin up drawings and models in a realistic setting.

With the onset of the development of gaming on social platforms in the virtual, many have begun to implement opportunities for user development. For instance, the group of designers and artists at Illusorr launched a virtual world (see Figure. 5) via the Sansar platform, where artists and designers share their work and host tutorials. Such a platform creates a global community despite the currently limited ability to travel.

The capabilities have begun to translate into various architectural and artistic displays and exhibitions, most notably during the COVID-19 pandemic due to the limitations on physical interaction [10]. As a result of the cancellation of the annual Burning Man festival, the event was instead held within a virtual world hosted on the AltSpace platform. This allowed for the designer and architect Arthur Manu-Mouri to display his amphitheater as well as a couple of past pavilions in a virtual manner for visitors to enjoy (see Figure. 5). Students have begun taking advantage of the social capability of the software and have begun to use the AltSpace as a platform for discourse of their projects. While student-generated VR experiences allow for a single user to view the model independently and require the transfer of large files, hosting projects within social platforms such as AltSpace allow for not only concurrent multi-viewer occupation and interaction but also conversations to occur within the digital environment.
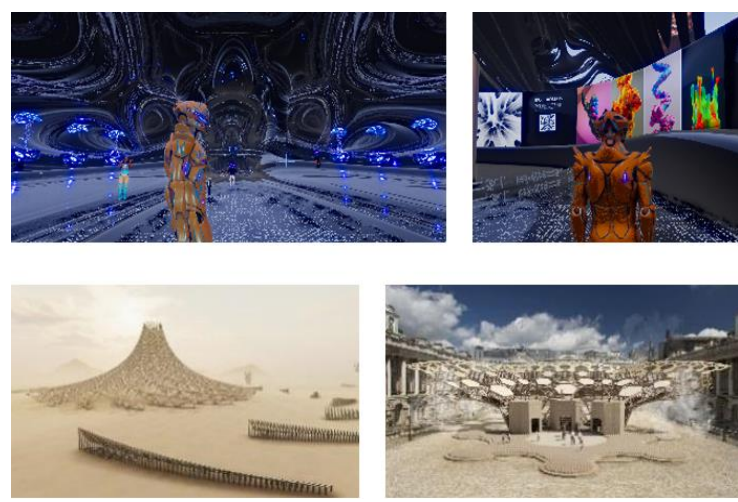

Figure 5. VR tour of the Illusorr world launch, with artist work on display (above), Pavilions at the 2020

Virtual Burning Man Festival via the AltSpace platform (below) 
In sharing virtual designs and experiences such as Illusorr and the virtual Burning Man festival in Altspace, the aspect of community has been brought to the digital world. The occupation and interaction within virtual art installation showcases the capabilities of VR use in design feedback, giving users the experience of the art without concerns of travel and cost. In addition to being more enjoyable than a two-dimensional video of the experience, observers become explorers and make a journey of their own around the design engaging in different perspectives that in turn become varied reactions and responses as feedback for designers.

\section{Extended Realities for Experiential Learning}

Due to the applied nature of construction and design, experiential learning, an augmentation of classroom-based education, is a critical component of architectural pedagogy. As design schooling encompasses a large variety of components, from conceptual design to detailing, costing, construction, and fabrication expertise, XR technologies are able to improve content delivery and better engage students. Through experiential learning, students are able to partake in on-site applications of their architectural knowledge and learn via hands-on participation, opportunities which are invaluable for students to gain a holistic understanding of the uses and overlaps of their learning.

Construction site visits serve as an instrumental method of architectural instruction where students are able to take their theoretical knowledge taught within coursework and understand how it manifests in the field. Students take a front seat to processes, assemblies, and the use of machinery they study, giving them insight into more practical perspectives.

Despite the value of such experiences, they are not always possible or available. While in the past extracurricular excursions to bring students to building sites were conducted annually at DAS, the restrictions imposed during the coronavirus pandemic restricted the possibilities of such visits. Although the limitations of personnel on site in combination with the immobility of students scattered across the globe prevented the conduction of such opportunities, XR technologies were able to provide alternate means of experience. As 360degree imagery was taken at previously conducted trips, professors were able to distribute these images to their students as alternative means of experience. Students were able to view these images via their mobile phones in order to simulate an in-person site visit in an experiential variation of an iVFT.

Mixed reality has also proven to be invaluable in expanding experiential learning both within and outside of curricular contexts. Previous to the acquisition of this technology, DAS students were frequently engaged to create design-build projects, which would require student teams to design, detail, fabricate, and construct art installations, pavilions, booths, and more for public exhibitions and festivals. These projects were invaluable to evolve students' understanding of logistics, project management, and realization of built work while simultaneously giving students a platform to express their ideas, contribute meaningfully to architectural discourse, and expand their portfolios of work. However, due to the complexity of the designs, students frequently encountered barriers and difficulties during the fabrication process, occasionally resulting in redesigns and wasted resources. Design-build projects at DAS are intended to engage a wide range of students, with upper years performing design and project management tasks while younger years aid in the fabrication, assembly, and installation processes. Thus, communication between groups often became challenging, requiring many sets of drawings and instructions, increasing mistakes and human error. These issues often interfered with the learning processes and impaired the projects' success in varying capacities. Despite such setbacks being fairly typical of projects within the industry, many issues prove to be extraneous for the students causing excessive stress and strain. The elimination of these challenges can lead to more healthy and successful learning environments. Through the implementation of MR into the fabrication process, many of these problems can be resolved.

The combination of the Hololens 2 and the software Fologram, architecture students were challenged to use MR technologies to reconstruct digitally modeled designs physically. Fologram, which also acts as a plug-in into the wide-spread software used by students to generate digital models, is able to connect the modeling software directly to the headset. This makes the digital model available for both viewing and editing within the holographic environment.

MR students are given the ability to view holographic assembly instructions, eliminating the need for complex 2D diagramming for 3D aggregations. This allows students to not only view their progress and successfully gauge accuracy, but also view their model within the real-world context.

In addition, students are able to edit parameters and placement of objects within their digital model from the headset itself, while seeing the projection into their immediate physical space. Students were challenged to experiment with a variety of forms that would be difficult to replicate in $3 \mathrm{D}$ from $2 \mathrm{D}$ orthographic instruction, including lattices and organic shapes (see Figure 6).

One particular benefit of MR is the collaborative possibilities the technologies initiate. For instance, despite there being a limited number of Hololens 2 headsets available for use by students for fabrication, 
other students without direct access to the headset are able to use AR alternatives on their mobile devices which are able to synchronize to the view from the headset. In this manner, all the participating students are able to engage in the XR experience and view the holographic guides, albeit with reduced accuracy.
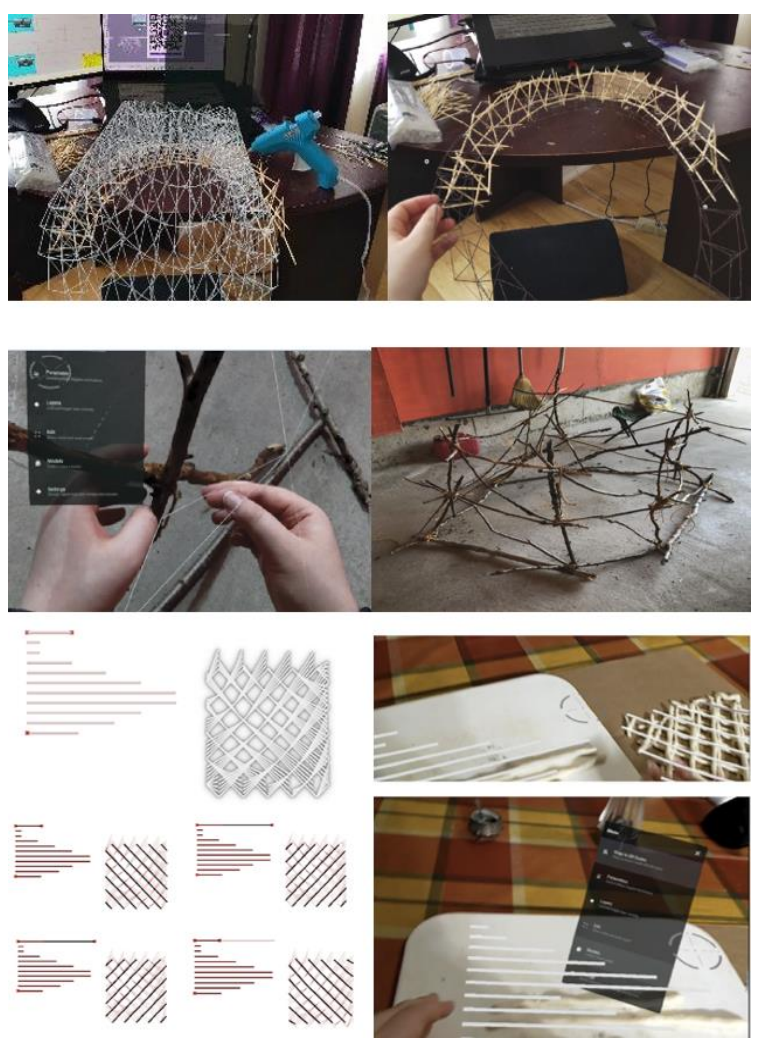

Figure 6. Student projects engaging MR via the Hololens 2 and Fologram, wood lattice construction at a small scale (above), large scale using found biomaterials (center), and with moldable clay-like forms (below)

The elimination of construction drawings and complex assembly instruction manuals makes the design-build process more accessible and less intimidating for students. [15]

Within experiential learning contexts, extended reality technologies can expand the capabilities of student experiences, improving accessibility and quality of investigative architectural education. Despite being in its infancy in terms of implementation within DAS, the case studies thus far have proven that MR technologies improve designbuild workflows and alleviate many extraneous issues students face within these extracurricular endeavors in order to guide the focus on the experimental learning itself. With the rising recognition of experiential learning as a critical component of architectural education, these technologies will continue to facilitate such opportunities for students.

\section{Discussion}

A number of software, outlined in Table 2, have been used within DAS in efforts to expand accessibility and engagement within architectural education. Each application of XR and corresponding software are able to provide a unique experience for users and vary the experience of unbuilt architectural spaces. From traditional 2-dimensional visualizations to now immersive technologies, architectural pedagogy has evolved to where students have the opportunity to live and learn in the architecture they create. The XR software chosen to be used for students were selected for their accessibility in pricing and computer resource utilization, balancing convincing real time graphics while requiring optimal hardware available at the institutions. The software listed in Table 2. showcase the several XR software implemented into the DAS academic curriculum encouraging students to visit their projects in a virtual environment rather than through a computer screen. With VR, the relevance in architectural pedagogy is found through lectured based instruction, design ideation, feedback, and experiential learning, as a versatile tool capitalizing on the freedom in using digital worlds as a means to design, present, and explore.

Furthermore, the compatibility of VR rendering software with $3 \mathrm{D}$ modelling software ensures a simple workflow when generating basic VR experiences. In recent years, the integration of adaptive feedback and interactivity have been catalyzed by positive statistical evidence of VR methods' such as iVFTs impact on improving students' content knowledge [9]. Navigable VR allows for more advanced experiences to be virtually simulated such that it gives the viewer greater control over their placement within these spaces through the use of responsive components. Similar to 360-degree static imagery, the accessibility of navigable VR on multiple platforms such as desktops and smartphones allow for its deployment and usage to be democratized among students and educators. Additionally, its ability to be superimposed onto multi-media installations offer opportunities for students to explore and compare formal and virtual environments. Interactive VR's popularity as a form of entertainment has ultimately led professionals and academics to leverage their capabilities and fidelity to be used as a learning tool.

The use of AR, it has a similar impact in architectural education as a tool that combines real and virtual information, most often used as a virtual overlay tool transforming the environment from the lens of a camera to responsive and immersive spaces. In lecture-based learning, AR is used as an assistant in GPS augmented learning while being a tool for combining virtual models and physical models in design ideation. In design discussions, AR is utilized 
to bring virtual models into the physical scale and imposed onto existing environments. As a visual aid, $\mathrm{AR}$ is able to enhance experiences in architectural pedagogy as a tool that blends virtual and reality.

Meanwhile, MR has been able to expand the possibilities within augmentation to allow for students to bridge gaps between their understanding of digital and physical space. This technology has the potential to accelerate complex design and fabrication endeavors and give students additional confidence when entering the workforce.

Table 2. Summary of XR software used at DAS for creation and ideation

\begin{tabular}{|c|c|c|c|c|}
\hline Software & $\begin{array}{l}\text { Software } \\
\text { Typology }\end{array}$ & XR Typology & Applications & $\begin{array}{l}\text { Benefits for } \\
\text { Architectural Pedagogy }\end{array}$ \\
\hline Yulio & Plug-in & 360-degree stills & $\begin{array}{l}\text { Panoramic } 360 \\
\text { visuals for } \\
\text { presentation }\end{array}$ & $\begin{array}{l}\text { Immersive design tool } \\
\text { for core studios }\end{array}$ \\
\hline Arch-App & $\begin{array}{l}\text { Standalone } \\
\text { Software }\end{array}$ & Interactive AR & $\begin{array}{l}\text { Site visits, Database, } \\
\text { Visualization }\end{array}$ & $\begin{array}{l}\text { Experiential technology } \\
\text { for history and theory- } \\
\text { based lecture courses }\end{array}$ \\
\hline Enscape & Plug-in & $\begin{array}{l}\text { Live navigable VR } \\
\text { environments }\end{array}$ & $\begin{array}{l}\text { Student-generated } \\
\text { navigable } \\
\text { environments }\end{array}$ & $\begin{array}{l}\text { Multisensory occupation } \\
\text { of VR environments, } \\
\text { animation fly through }\end{array}$ \\
\hline Twinmotion & $\begin{array}{l}\text { Plug-in, } \\
\text { Standalone } \\
\text { Software }\end{array}$ & $\begin{array}{l}360 \text {-degree stills } \\
\text { and videos }\end{array}$ & $\begin{array}{l}\text { Social and occupiable } \\
\text { environments }\end{array}$ & $\begin{array}{l}\text { Multisensory occupation } \\
\text { of VR environments, } \\
\text { animation fly through }\end{array}$ \\
\hline Mindesk VR & Plug-in & $\begin{array}{l}\text { Live navigable VR } \\
\text { environments }\end{array}$ & $\begin{array}{l}\text { Digital experiences of } \\
\text { construction sites and } \\
\text { processes }\end{array}$ & $\begin{array}{l}\text { VR-based digital } \\
\text { modeling }\end{array}$ \\
\hline $\begin{array}{l}\text { Gravity } \\
\text { Sketch }\end{array}$ & $\begin{array}{l}\text { Standalone } \\
\text { Software on } \\
\text { headsets }\end{array}$ & $\begin{array}{l}\text { Live navigable VR } \\
\text { environments }\end{array}$ & $\begin{array}{l}\text { 3D Modelling, Multi- } \\
\text { user design }\end{array}$ & $\begin{array}{l}\text { Collaborative digital } \\
\text { modeling, VR-based } \\
\text { visualization }\end{array}$ \\
\hline$A R I D D$ & $\begin{array}{l}\text { Standalone } \\
\text { Software }\end{array}$ & Interactive AR & $\begin{array}{l}\text { 3D Modelling, } \\
\text { Visualization }\end{array}$ & $\begin{array}{l}\text { Experiential learning, } \\
\text { Iterative scaled } \\
\text { modelling }\end{array}$ \\
\hline AltSpace & $\begin{array}{l}\text { Standalone } \\
\text { Software }\end{array}$ & $\begin{array}{l}\text { Live navigable VR } \\
\text { environments }\end{array}$ & $\begin{array}{l}\text { Multi-User } \\
\text { interactive viewing } \\
\text { platform }\end{array}$ & $\begin{array}{l}\text { Collaborative and } \\
\text { multisensory occupation } \\
\text { of VR environments }\end{array}$ \\
\hline Spatial.io & $\begin{array}{l}\text { Standalone } \\
\text { software on } \\
\text { headsets and } \\
\text { mobile devices }\end{array}$ & $\begin{array}{l}\text { Live navigable VR } \\
\text { environments, } \\
\text { Interactive } \mathrm{AR}\end{array}$ & $\begin{array}{l}\text { Multi-User } \\
\text { interactive viewing } \\
\text { platform }\end{array}$ & $\begin{array}{l}\text { Collaborative design } \\
\text { review, incorporation of } \\
\text { three-dimensional virtual } \\
\text { supplementation in } \\
\text { virtual critiques }\end{array}$ \\
\hline Fologram & $\begin{array}{l}\text { Plug-in, } \\
\text { Standalone } \\
\text { Software on the } \\
\text { Hololens } 2\end{array}$ & $\begin{array}{l}\text { Interactive MR } \\
\text { overlays bridging } \\
\text { the digital models } \\
\text { to the physical } \\
\text { realm }\end{array}$ & $\begin{array}{l}\text { Fabrication and } \\
\text { assembly guides for } \\
\text { student design-build } \\
\text { projects. }\end{array}$ & $\begin{array}{l}\text { Easy of fabrication of } \\
\text { complex geometries, } \\
\text { improved project } \\
\text { management, enhanced } \\
\text { collaboration during } \\
\text { fabrication }\end{array}$ \\
\hline
\end{tabular}

\section{Conclusion}

The VR, AR, and MR technologies are most often associated with entertainment purposes, they have also undergone rapid growth and implementation within both academic and professional contexts. It has allowed educators to employ more elaborate design projects and experiences while optimizing the readily available resources provided by the institution. In the case of remote learning, VR, AR, and MR have become a fundamental tool for design thinking and iteration in the studio curriculum as viewers are able to fully immerse themselves in conceptual and existent spaces despite where they are physically. While it may not be a completely seamless transition into a virtual environment, the vast amount of information and resources available online combined with the ubiquity of mobile devices makes the three uses of digital realities an accessible tool. Through the use of the three XR typologies in the period of remote 
learning, DAS has explored various technologies to build upon methods of architectural education, discourse, and exploration, finding several avenues in which the XR applications can be capitalize.

In light of the COVID-19 pandemic, XR technologies have also served as a tool to enable creative discourse between educators and students in the context of studio courses. Current uses of VR, AR, and MR, have connected students and institutions across the world, bringing design explorations and discussion to the digital forefront manifested by the social distancing of the pandemic. Furthermore, XRs widespread usage in academia is evident in multiple areas of study beyond design and architecture such as medicine, fine arts, and forensic science [16].

The growing adoption of XR is expanding, which prompts questions of additional methodologies and uses using VR, and AR with many possibilities currently appearing in uses of MR. MR as an extension of VR and AR proves to be invaluable in the AEC industry as a tool that can blend the virtual CAD model world with construction on site, while in realtime. While still in a phase of nascency, MR is being developed to improve methods of construction and ease coordination efforts in design projects. By being able to go beyond mere visualization and into serving as an aid to fabrication, MR paves the way for the implementation of future construction technologies. The usage of VR, AR, and $\mathrm{MR}$ as a robust pedagogical tool is an emerging practice within the realm of architecture; its adoption into the mainstream is favorable in many industries due to the efficacy of the medium to communicate, evaluate, and virtualize experience.

\section{References}

[1] Christou, C. (2010). Virtual reality in education. Affect. Interact. Cogn. Methods E-Learning Des. Creat. an Optim. Educ. Exp., no. February, pp. 228-243, DOI: 10.4018/9781-60566-940-3.ch012.

[2] Hui, V. Estrina, T. Zhou, G. Lee, S. and Kinuthia V. (2020). Architectural Accessibility and Pedagogy via Virtual Reality, in HUIC ASHE Conference Proceedings, pp. 1-13.

[3] Estrina, T. and Hui, V. (2020). Extended Realities as Methods of Representation within Architectural Pedagogy.

[4] Wang, P. Wu, P. Wang, J. Chi, H. L. and Wang, X. (2018). A critical review of the use of virtual reality in construction engineering education and training. Int. J. Environ. Res. Public Health. Vol. 15, no. 6, DOI: 10.3390/ijerph15061204.

[5] Ruiz, R. Weghorst, S. Savage, J. Oppenheimer, P. Iii, T. F. and Dozal, Y. (2002). Virtual Reality for Archeological Maya Cities. UNESCO World Herit. Cent. Conf.
[6] Ishii H. et al., (2002). Augmented urban planning workbench: Overlaying drawings, physical models and digital simulation," Proc. - Int. Symp. Mix. Augment. Reality, ISMAR-2002, pp. 203-214. DOI: 10.1109/ISM AR.2002.1115090.

[7] Jahn, G., Newnham, C., \& van der Berg, N., \& Iraheta, M. \& Wells, J., (2019). Holographic Construction.

[8] R. G. Alvarado and T. Maver, "Virtual Reality in Architectural Education: Defining Possibilities," ACADIA Q., vol. 18, no. 4, pp. 97-99, 1999, http://cum incad.scix.net/cgi-bin/works/Show?_id=4d95\&sort=DEF AULT\&search=virtualreality\&hits $=2442$ (Access Date: 15 May 2021).

[9] Mead, C. Buxner, S. Bruce, G. Taylor, W. Semken, S. and Anbar, A. D. (2019). Immersive, interactive virtual field trips promote science learning. J. Geosci. Educ., vol. 67, no. 2, pp. 131-142, Apr. DOI: 10.1080/10899995.201 9.1565285

[10] Adhanom, T. (2020). WHO Director-General's opening remarks at the media briefing on COVID-19 (11 March 2020). World Health Organization. https://www.w ho.int/director-general/speeches/detail/who-director-genera 1-s-opening-remarks-at-the-media-briefing-on-covid-19-11 -march-2020 (Access Date: 20 May 2021).

[11] Hui, V. Townsend, S. Gantous, S. and McCarthy, G. (2013). Arch App | An Application for Architectural Design Pedagogy.

[12] Hill, D. (2019). How Virtual Reality Impacts the Landscape Architecture Design Process at Various Scales, All Grad. Theses Diss. https://digitalcommons.usu.edu/et d/7519 (Access Date: 12 April 2021).

[13] Di Gironimo G. and Lanzotti, A. (2009). Designing in VR. Int. J. Interact. Des. Manuf., Vol. 3, no. 2, pp. 51-53, , DOI: $10.1007 / \mathrm{s} 12008-009-0068-6$.

[14] Hui, V., Compeau, M., Pu, K. and Arabian, T. (2013). Design In Hand | A Pedagogical Application Of Augmented Reality In Design Development, in INTED2013 Proceedings, pp. 5987-5996.

[15] Jahn, G., Newnham, C., van den Berg, N. and Beanland, M. (2018). Making in Mixed Reality," Acadia, January, pp. 88-97, http://papers.cumincad.org/cgi-bin/ works/BrowseTreefield=seriesorder=AZ/Show acadia 18 _88 (Access Date: 16 April 2021).

[16] C. Tawhai, (2017). Immersive $360^{\circ}$ Video For Forensic Education. Perth. 